\title{
COMPUTER ANALYSIS OF PHYSIOLOGIC SIGNALS IN A CARDIOVASCULAR RESEARCH LABORATORY
}

\author{
Saul E. Greenhut, Jong W. Lee, Joseph C. Monsur and \\ JOHN M. NICKLAS \\ Departments of Internal Medicine, Electrical Engineering and Computer Science, \\ The University of Michigan, Ann Arbor, MI 48109-0366, U.S.A.
}

(Received 18 April 1990; in revised form 13 September 1990; received for publication 24 September 1990)

\begin{abstract}
A comprehensive computer program which provides immediate computation and feedback has been developed for data acquisition and analysis of signals in a cardiovascular animal laboratory. The system is based on a microcomputer equipped with analog-to-digital converter and supports function modules which digitize, filter, and differentiate up to 8 simultaneously sampled cardiovascular signals. The program detects, analyses, and plots incoming and averaged beats. Beat-by-beat signal averaging for each channel is performed and cardiac cycles are partitioned automatically. For each cardiac and average cycle the amplitude at 6 physiologic fiducial markers are measured and derived calculations are made. Channel vs channel plots and loop area measurements are also computed and displayed. The computer algorithms have been shown to give accurate, precise, and reproducible results when tested on canine cardiovascular data. Also, it has been demonstrated that signal averaging is an appropriate analysis technique for cardiovascular signals.
\end{abstract}

Computer Signal processing Signal averaging Cardiovascular signal analysis Animal laboratory

\section{INTRODUCTION}

Computer-aided data collection and analysis is a valuable tool in laboratory research. Besides saving a significant amount of time and effort over manual analysis of analog output on the part of the researcher, on-line signal sampling and processing can provide immediate feedback to the scientist allowing a type of servo-control of an experimental protocol. In addition, manual analysis which is not blind may cause results to be coerced toward the direction of the researcher's hypotheses. Automated data analysis is reproducible, so that user bias and variability are eliminated. Thus, such a computer system can greatly expedite research efforts by improving experimental efficiency and accuracy.

Although computer analysis systems have been individually developed for many laboratories, we present a new, flexible, and efficient program based on commonly available and inexpensive hardware for general use in any cardiovascular laboratory. The program samples and analyses eight physiologic signals. Signal processing includes averaging, filtering, differentiating, and determining end-diastole, end-systole, beginejection, and begin-filling automatically on beat-by-beat and averaged data. Data analysis, performed on either incoming or averaged beat data, includes calculating waveform parameters, single variable plotting, and two variable $(X-Y)$ plotting. The flexibility of this system is evident in the wide array of possible channel designations, the variety of channel selections for cardiac cycle and signal average triggering, the options available in plotting formats, and the ability to override and specify new program default parameters.

\section{MATERIALS AND METHODS}

The system is based on an IBM PC (PC, XT, or AT) or compatible personal computer with 640 kbytes of random access memory (RAM). The software, written in C program- 


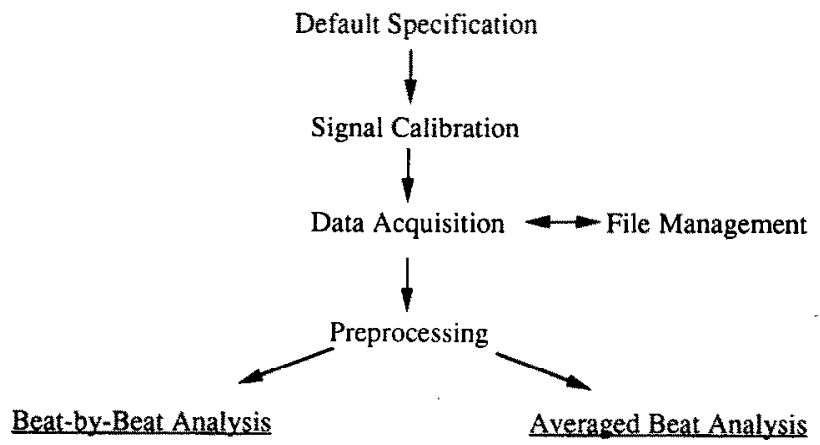

\footnotetext{
- Cardiac cycle analysis

- Parameter calculation

- File Managemen

- Signal Plotting

- Single channel plot

- Channel vs. channel $(X-Y)$ plot

- $\mathrm{X}$-Y plot area measurement
}

Fig. 1. Functionai analysis of the cardiovascular analysis computer system described in text.

ming language, will work with a variety of graphics drivers. An IBM compatible dot-matrix graphics printer allows hard copies of data and screen plots to be obtained.

Data are acquired using the Tecmar Lab Master analog-to-digital (A/D) system (Scientific Solutions, Inc., Solon, $\mathrm{OH}$ ). The Tecmar board provides 16 channels of either unipolar or bipolar input signals which also can be configured for 8 true differential inputs. The maximum sampling frequency of the $A / D$ subsystem is $50 \mathrm{kHz}$ and the resolution is 12 bits per sample. For our system, the board is configured such that the onboard clock controls sampling and the input signal range is set to $\pm 5 \mathrm{~V}$.

The program processes a maximum of 8 channels of analog data concurrently sampled for up to 5000 digital points per channel per sampling period (e.g. $20 \mathrm{sec}$ at $250 \mathrm{~Hz}$ ). Input signals are assumed to include either left ventricular pressure (LVP), the derivative of LVP $(\mathrm{dP} / \mathrm{dt})$ or the electrocardiogram (ECG), since at least one of these waveforms is necessary for cardiac cycle analysis. In order to provide the necessary timing signal if beat averaging is desired, an ECG or a waveform of similar cycle timing and morphology must be included as one of the signals.

\section{THE PROGRAM}

Figure 1 shows the functional organization of the program. Each item is described in detail below.

Default specification. The researcher may designate default settings which completely specify the protocol for a series of experiments. The user may store the default information to a disk file which may be recalled for a future experiment. Table 1 lists the items specified on the default screen and their original values.

Signal calibration. Low and high calibration values are acquired on all desired channels and the corresponding quantities in appropriate physical units are entered by the investigator. All results are then normalized according to the mapping of the sampled signals in $\mathrm{mV}$ to the correct units for each channel. This calibration information may be saved to a disk file for analysis of the data offline. Also displayed is the theoretical precision of each channel in measured units (e.g. $\mathrm{mmHg}$ ), given the calibration signal range and the $A / D$ quantization error.

Data acquisition. All eight channels are acquired concurrently in multiplexed fashion. Data may be sampled at varying rates and for varying durations. Specific analysis routines are available for LVP, arterial pressure, venous pressure, atrial pressure, 
derivative of LVP $(\mathrm{d} P / \mathrm{d} t)$, second derivative of LVP $\left(\mathrm{d}^{2} P / \mathrm{d} t^{2}\right)$, tissue segment length, tissue wall thickness, volume diameter, blood flow, and ECG, although any physiologic signal may be acquired and analysed using a generic channel. Once the data are received, a plot of the eight sampled signals moving in time may be displayed on the screen for visual verification of the sampling process.

File management. Several types of data files are supported by the program. The first type stores the digitized data as received directly from the $A / D$ and signal calibration information. These data may be recovered at a later date for analysis.

Two data file types for individual beat analysis parameters are available. The first file stores the measured and calculated values for each beat. The second file stores mean parameters of all beats for each sampling period. By saving the mean values as an entry for each sampling period, a table is formed which provides a complete history of the experimental protocol. If averaged data analysis is desired, a corresponding file which stores average beat parameters also forms a summary of the experiment. The data stored in the analysis files may be conveniently recalled and printed.

\section{Preprocessing}

1. Filtering. This feature is available for use in several possible applications. The filter is a linear phase finite impulse response (FIR) band-pass filter $[1,2]$. The filter uses a Hamming window and $0.1 f_{\mathrm{s}}$ coefficients, where $f_{\mathrm{s}}=$ sampling rate. The user specifies the low and high cut-off frequencies. Therefore, the filter may serve as a low-pass, high-pass, or band-pass type.

The band-pass filter described has several applications when used on cardiovascular

Table 1. Program default items and original values

\begin{tabular}{|c|c|}
\hline Item on default screen* & Original default value $\dagger$ \\
\hline Specification of channel designations $1-10$ & $\begin{array}{l}\text { 1. Left ventricular pressure } \\
\text { 2. } \mathrm{d} P / \mathrm{d} t \\
\text { 3. Atrial pressure } \\
\text { 4. Arterial pressure } \\
\text { 5. Blood flow } \\
\text { 6. Segment length-1 } \\
\text { 7. Segment length-2 } \\
\text { 8. Electrocardiogram } \\
\text { 9. Computer derived } \mathrm{d} P / \mathrm{d} t \\
\text { 10. Computer derived } \mathrm{d}^{2} P / \mathrm{d} t^{2}\end{array}$ \\
\hline Data disk drive & A \\
\hline Printer port or file to store data & $1 \mathrm{ptl}$ \\
\hline Sampling rate & $250 \mathrm{~Hz}$ \\
\hline Sampling duration & $10 \mathrm{sec}$ \\
\hline End-diastolic trigger channel & 8 \\
\hline End-systolic trigger channel & 9 \\
\hline Msec before $\mathrm{d} P / \mathrm{d} t_{\min }$ & $10 \mathrm{msec}$ \\
\hline$\%$ after end-diastole (if ECG channel used) & $50 \%$ \\
\hline Begin-ejection trigger channel & 9 \\
\hline Msec after $\mathrm{d} P / \mathrm{d} t_{\max }$ & $10 \mathrm{msec}$ \\
\hline Begin-filling trigger channel & 1 \\
\hline MmHg above LVEDP $\ddagger$ & $0 \mathrm{mmHg}$ \\
\hline ECG trigger channel & 8 \\
\hline QRS trigger polarity & Up \\
\hline$\%$ of average beat displayed before end-diastole & $20 \%$ \\
\hline Display begin-ejection and begin-filling data & Yes \\
\hline Convexity fraction threshold & 0.70 \\
\hline $\begin{array}{l}\text { Automatic computer calculation of } \mathrm{d} P / \mathrm{d} t \\
\text { Low-pass cut-off frequency for } \mathrm{LVP}\end{array}$ & $\begin{array}{l}\text { Yes } \\
50 \mathrm{~Hz}\end{array}$ \\
\hline Automatic computer calculation of $\mathrm{d}^{2} P / \mathrm{d} t^{2}$ & Yes \\
\hline Low-pass cut-off frequency for $\mathrm{d}^{2} P / \mathrm{d} t^{2}$ & $30 \mathrm{~Hz}$ \\
\hline Default filename & default.dft \\
\hline
\end{tabular}

* Items which may be specified on the default screen.

$\dagger$ Original default values corresponding to the respective item in the left column.

$\ddagger$ Left ventricular end-diastolic pressure. 
signals. As a low-pass filter it can be used to eliminate high frequency signal noise (e.g. myopotential noise), while as a high-pass filter it can eliminate baseline wander. The filter can also be used to eliminate unwanted high or low frequency waveforms from a signal. For example, pacemaker generator pulses may be eliminated from, or isolated in, an ECG complex by specifying the appropriate cut-off frequencies.

2. Differentiation. In the event an analog $\mathrm{d} P / \mathrm{d} t$ signal is not available, a digitally computed $\mathrm{d} P / \mathrm{d} t$ is desired, or a derivative of another signal is requested, a routine for numerically differentiating any channel is provided. If an LVP signal is available, the program may be directed to calculate $\mathrm{d} P / \mathrm{d} t$ and $/$ or $\mathrm{d}^{2} P / \mathrm{d} t^{2}$ immediately following signal digitization or after the recall of a data file. The results are stored in two supplementary channels 9 and 10. Before this differentiation, LVP is low-pass filtered with a frequency cut-off chosen by the user (default $=50 \mathrm{~Hz}$ ) in order to reduce the noise in the resultant derivatives (see below). If $\mathrm{d}^{2} P / \mathrm{d} t^{2}$ is calculated, the result is filtered at a high frequency cut-off also chosen by the user (default $=30 \mathrm{~Hz}$ ).

The differentiation is obtained using a 3-point Lagrange derivative approximation [3]:

$$
\frac{\mathrm{d} f\left(t_{i}\right)}{\mathrm{d} t}=\frac{f\left(t_{i+1}\right)-f\left(t_{i-1}\right)}{2 h}
$$

wherc $f\left(t_{i}\right)$ is a discrete sample value of a continuous function $f(t)$ and $h$ is the sampling interval.

Because numerical differentiation introduces approximation error which appears as noise, the input signal must be low-pass filtered before differentiation or the derivative result must be low-pass filtered at a suitable cut-off frequency. The FIR filter described above is used for this purpose.

Beat-by-beat analysis vs averaged beat analysis. Following preprocessing, the laboratory investigator has a choice of two types of analysis. Beat-by-beat analysis preforms cardiac cycle analysis and parameter calculations on each individual beat, while averaged signal analysis examines a single mean beat. For either type of analysis it is possible for the user to specifically eliminate undesirable beats, while averaged beat analysis also automatically discards abnormal cycles based on the trigger signal selected. The remaining discussion assumes the ECG serves as the beat averaging signal trigger, although the operations described may be applied to any waveform.

Signals are averaged after waveforms are aligned using the QRS complex of the ECG as a fiducial point. QRS complex position is determined using a convexity operator (CON) as defined by:

$$
\operatorname{CON}=q r s_{-} \text {pol } \cdot((2 \operatorname{ecg}[i])-e c g[i-12 \mathrm{msec}]-e c g[i+12 \mathrm{msec}])
$$

where ecg[i] is the ECG signal amplitude in $\mathrm{mV}$ at time $i$ msec and qrs_pol is a user specified value remaining constant for an entire sampling period. Qrs_pol is set to 1 by the user for normal QRS complexes of upward polarity and set to -1 for normal QRS of downward polarity. Thus, the magnitude of the convexity is large for sharp and high amplitude waveforms, while it is smaller for slowly changing and low amplitude waveforms. The user specifies the polarity of normal QRS complexes, in order to increase the specificity of the detection algorithm.

A convexity threshold, defined as a fraction of the maximum convexity, is determined for each sampled segment of data. The user may redefine this fraction (default $=0.7$ ). Portions of the data greater than the predefined threshold for qrs_pol=1 and less than the threshold for $q r s_{-} p o l=-1$ are designated as QRS complexes and a maximum or minimum amplitude search in this region locates the actual waveform peak. The convexity operator and $\mathrm{R}-\mathrm{R}$ interval criteria use morphology and timing to discriminate 
normal beats from premature, escape, and aberrant conductions which are discarded. In addition, if the $\mathrm{R}-\mathrm{R}$ interval is abnormal, the previous and following cardiac cycles are eliminated from analysis, due to possible hemodynamic variability. Mean signals are determined for all ten channels simultaneously, after which cardiac cycle analysis and parameter calculations may be performed using identical criteria as defined for individual beat analysis (see below).

Cardiac cycle analysis. After data collection is completed, the program locates automatically the following ventricular cycle events on a sequence of unaveraged beats or on a single mean beat (depending upon the analysis selected).

1. End of diastole. End-diastole is the end-point of myocardial relaxation, just prior to the onset of contraction (systole). The program may be specified to use either the ECG or $\mathrm{d}^{2} P / \mathrm{d} t^{2}$ to locate end-diastole. If the ECG is selected, the peak of the QRS complex, determined using the convexity operation described above, is used to mark this event. If $\mathrm{d}^{2} P / \mathrm{d} t^{2}$ is used, $\mathrm{d} P / \mathrm{d} t_{\max }$ is first located. One-hundred msec prior to $\mathrm{d} P / \mathrm{d} t_{\max }$ a forward search is initiated on $\mathrm{d}^{2} P / \mathrm{d} t^{2}$ until an upward peak is located whose amplitude is greater than $\left(\mathrm{d}^{2} P / \mathrm{d} t_{\max }^{2}\right) / 2$. Therefore, the first positive $\mathrm{d}^{2} P / \mathrm{d} t^{2}$ peak of each cycle above threshold is called end-diastole. The peak is found by using two consecutive slopes, the first of which must be greater than zero and the second less than zero.

2. End of systole. End-systole is defined physiologically as the time at which the heart has completely contracted, just prior to the beginning of relaxation (diastole). The endsystolic point is determined by the program using the negative peak of $\mathrm{d} P / \mathrm{d} t$. This $\mathrm{d} P / \mathrm{d} t_{\min }$ minus a user specified number of msec (default $=10 \mathrm{msec}$ ) is defined as end-systole and corresponds to the point at which LVP begins its steep negative slope following a plateau. A $\mathrm{d} P / \mathrm{d} t$ signal, acquired directly from analog instrumentation or digitally from LVP by the computer, is required for precise placement of end-systole. In lieu of a $\mathrm{d} P / \mathrm{d} t$ signal, the ECG may be selected to locate the end of systole. In this case, end-systole is placed a percent $R-R$ interval distance (default $=50 \%$ ) from the previous end-diastole.

3. Beginning of ejection. Begin-ejection occurs when blood is first ejected from the ventricles into the aortic and pulmonary arteries. This occurs when pressure in the ventricles exceeds that in the arteries, causing the semilunar valves to open. The program defines begin-ejection as a user specified number of $\mathrm{msec}$ (default $=10 \mathrm{msec}$ ) following maximum $\mathrm{d} P / \mathrm{d} t$.

4. Beginning of filling. Begin-filling is the start of ventricular filling which occurs at the end of isovolumic relaxation. The pressure in the atria becomes greater than that in the ventricles causing the atrioventricular (AV) valves to open. Begin-filling is determined by locating the point, following $\mathrm{d} P / \mathrm{d} t_{\min }$, at which LVP first becomes less than or equal to the previous left ventricular end-diastolic pressure (LVEDP). The user may designate that begin-filling be situated at a time at which the amplitude is above or below LVEDP by a specified number of $\mathrm{mmHg}$.

End-diastole, end-systole, begin-ejection, and begin-filling are displayed graphically and can be corrected by the program operator if found to be in error. Figure 2 shows a beat-by-beat display of $L V P$ and $\mathrm{d} P / \mathrm{d} t$ with computer-derived fiducial marks overlaid upon the digitized signals. Figure 3 shows an averaged beat representation of 8 channels, also with computer-derived physiologic markers.

Parameter calculation. Once the pertinent fiducial marks are located, many waveform parameters are calculated.

1. Waveform amplitudes. The amplitude at end-diastole, end-systole, begin-ejection, and begin-filling, and the maximum and minimum amplitude of each channel are recorded for each beat and the averaged beat.

2. Intervals. The interval between end-diastolic and end-systolic points, adjacent enddiastolic points and heart rate (as calculated from the $\mathrm{R}-\mathrm{R}$ interval) are determined. 


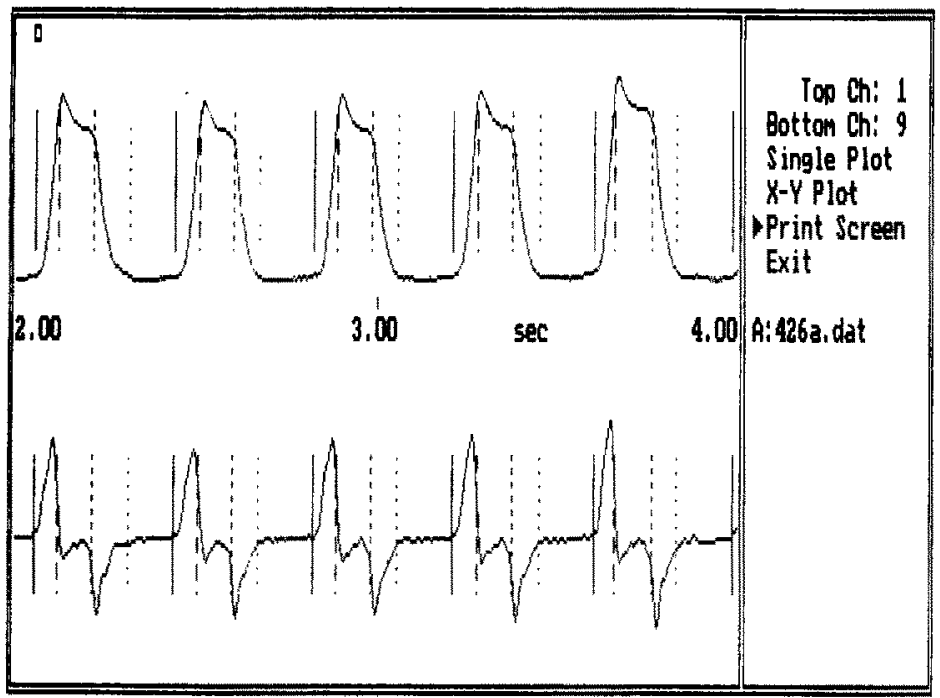

Fig. 2. The left ventricular pressure signal (top) and digitally derived $\mathrm{d} P / \mathrm{d} t$ (bottom). Computer derived, automatically placed, fiducial marks reflecting (from left for each beat): end-diastole (solid line), begin-ejection, end-systole, and begin-filling are superimposed on the tracings.

3. Percent change. The percent change (PC) reported is computed as:

$$
\operatorname{PC}(\%)=\frac{S_{\text {ed }}-S_{\text {es }}}{S_{\text {ed }}} \cdot 100
$$

where $S_{\text {ed }}$ and $S_{\text {es }}$ are the signal levels at end-diastole and end-systole. Percent change is denoted as segment shortening and diameter shortening, if tissue segment length and volume diameter were selected as channel designations. Also, $-\mathrm{PC}$ is reported as wall thickening for tissue wall thickness channels.

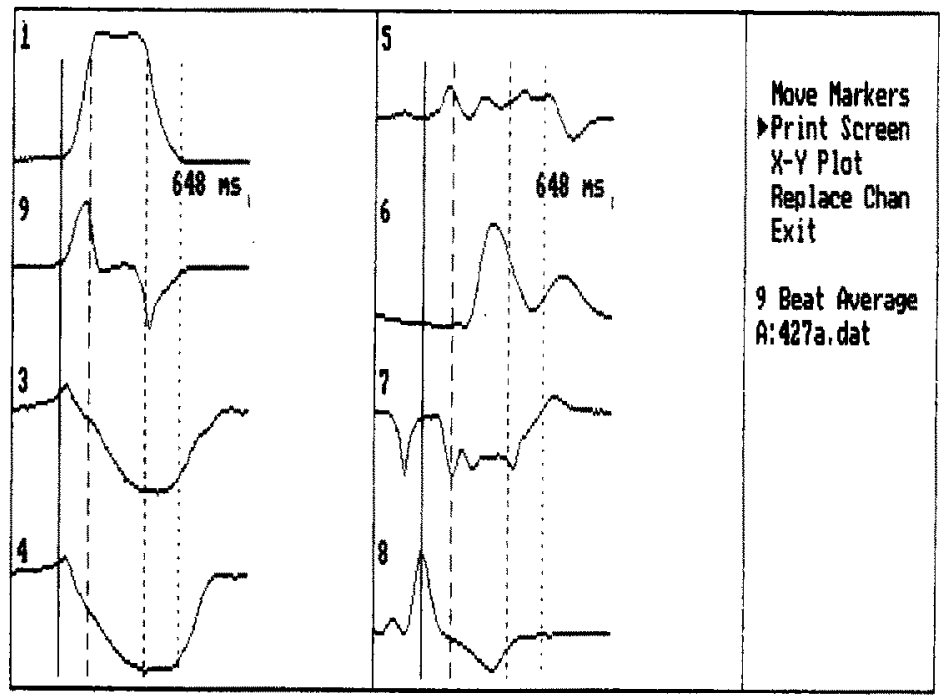

Fig. 3. Computer monitor display of averaged beat data from eight channels. Left ventricular pressure (channel 1), dP/dt (channel 2), myocardial segment length (channels 3 and 4), left atrial pressure (channel 5), carotid arterial pressure (channel 6), circumflex coronary arterial fow (channel 7), and ECG (channel 8) are shown. On the right, the display indicates that nine beats were averaged to yield the resulting plots. The solid, vertical line represents the computer derived location of end-diastole, while the remaining lines from left to right indicate beginejection, end-systolic, and begin-filling positions. 
4. Relaxation constant (T). That left ventricular pressure decay during isovolumic relaxation can be approximated by a monoexponential function was first determined by Weiss et al. [4]. More recently, it has generally been proven [5-7] that a monoexponential curve which accounts for possible shifts in the pressure curve is the best model describing the course of relaxation:

$$
P(t)=P_{0} \mathrm{e}^{-\mathrm{k} t}+P_{\mathrm{B}}
$$

where $P(t)$ is left ventricular pressure at time $t, P_{\mathrm{B}}$ is the asymptotic pressure at $t=\infty, P_{0}$ represents the initial value of pressure with respect to $P_{\mathrm{B}}, \mathrm{k}$ is a constant. The time constant of left ventricular isovolumic relaxation is:

$$
T=\frac{1}{\mathrm{k}}
$$

where $\mathrm{k}$ is from equation (4). $T$ is defined as the time needed for the pressure at $t=0$ to decline $1 / \mathrm{e}(36.8 \%)$ of the distance to its asymptotic value.

The program calculates the relaxation constant using a method described by Craig $e t$ al. [7]. Their technique assumes the monoexponential plus baseline shift model described by equation (4). The derivative of equation (4) is calculated which eliminates $P_{\mathrm{B}}$.

$$
\frac{\mathrm{d} P}{\mathrm{~d} t}=-\mathrm{k} P_{0} \mathrm{e}^{-\mathrm{k} t}
$$

Next, the natural logarithm of equation (6) is taken which yields:

$$
\ln \frac{\mathrm{d} P}{\mathrm{~d} t}=-\mathrm{k} t+\ln \left(\mathrm{k} P_{0}\right)
$$

Equation (7) is of the form of a line which permits a least-squares linear regression technique to be applied to the points representing equation (7) for calculating the slope k. Then $T$ is found via equation (5).

The range of LVP data points used in the calculation of $T$ begins at $\mathrm{d} P / \mathrm{d} t_{\min }$ and ends at the point which marks the beginning of filling. If fewer than 5 data points are included in this range, $T$ is not calculated due to the excessive approximation error inherent with such a small sample size.

5. Averages. The mean of all beat-by-beat parameters are calculated and represents a feature averaging, as opposed to signal averaging, of the data. Also, point-by-point time averages of arterial pressure, venous pressure, atrial pressure, and blood flow are calculated.

Figure 4 shows a sample print-out of beat-by-beat data for a numerically derived $\mathrm{d} P / \mathrm{d} t$ signal from a $10 \mathrm{sec}$ sampling interval. Figure 5 shows the print-out of an averaged data summary file which gives a complete record of an experiment.

Signal plotting. Individual channels of averaged and unaveraged data with fiducial markers for end-diastolic, end-systolic, begin-ejection, and begin-filling points can be displayed with beat parameters for visual confirmation. Computer generated markers may be manually adjusted if found to be in disagreement with operator determined locations and individual beats may be manually deleted if necessary. Averaged beat scaled plots with pertinent calculations, the scaling of which may be adjusted by the investigator, are also available. As an example of this display, Fig. 6 shows a carotid arterial average waveform with beat markers and parameters.

The $X-Y$ analysis of any two channels, averaged or not, may also be plotted. The operator specifies two channels and the time interval over which the $X-Y$ plot is to be 


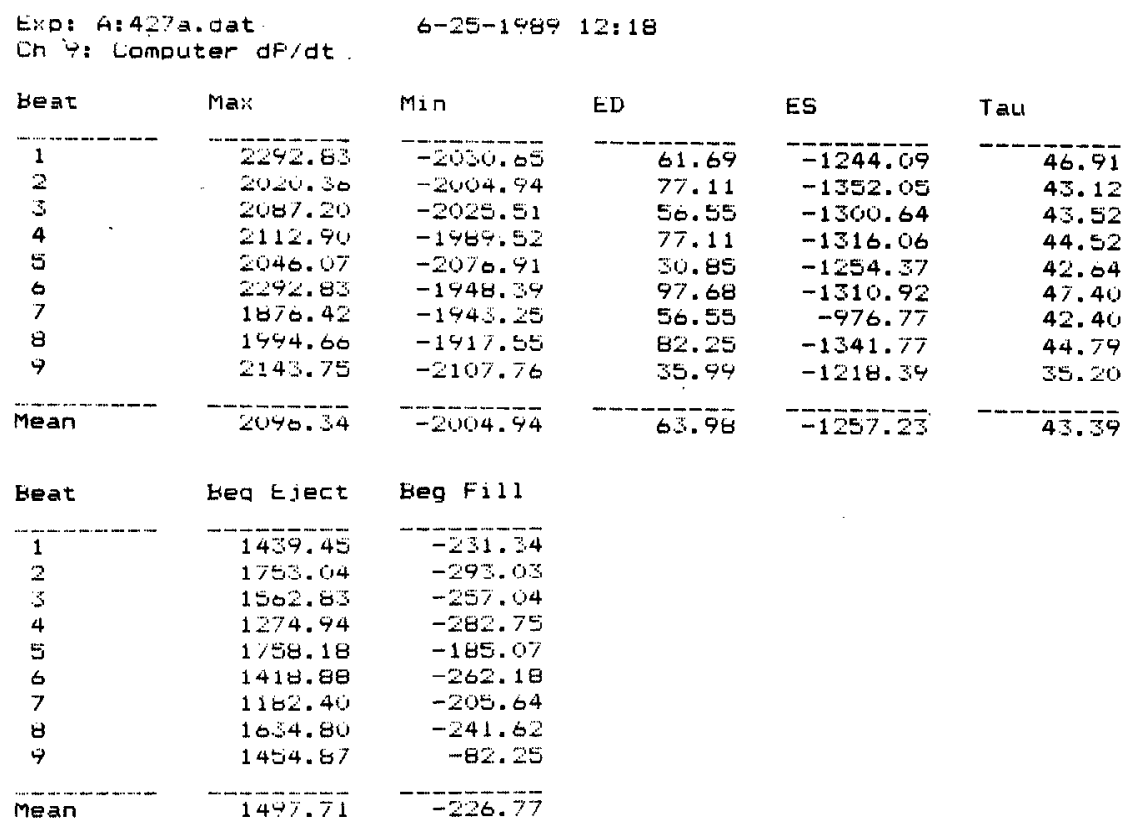

Fig. 4. Computer print-out of beat-by-beat, digitally derived $\mathrm{d} P / \mathrm{d} t$ values. The top line indicates the experimental data file, followed by the date and time. Below, the channel number (9) and channel type (computer $\mathrm{d} P / \mathrm{d} t$ ) are indicated. Abbreviations are as follows: Beat-current beat seqeuntial number, Max - maximum $\mathrm{d} P / \mathrm{d} t$ amplitude over current beat, Min-miniumum $\mathrm{d} P / \mathrm{d} t$ amplitude, ED-amplitude of $\mathrm{d} P / \mathrm{d} t$ at end-diastole, ES-amplitude of $\mathrm{d} P / \mathrm{d} t$ at end-systole, Tau-ventricular isovolumic relaxation time constant in msec, Begin eject-amplitude of $\mathrm{d} P / \mathrm{d} t$ at begin-ejection, Begin fill-amplitude of $\mathrm{d} P / \mathrm{d} t$ at begin-filling, Mean-mean average of values in that column. All $\mathrm{d} P / \mathrm{d} t$ amplitudes are in $\mathrm{mmHg} / \mathrm{sec}$. Note: each channel displays beat-by-beat parameters appropriate for its type.

drawn. Therefore, a series of cycle loops, one cycle loop or certain phases of a single loop may be plotted. Axes scaling X-Y plotting can be operator defined or automatically scaled and centered by the program. Any plot may be printed to an IBM compatible dotmatrix graphics printer using a graphics screen dump module included as part of the program.

Two variable, pressure vs volume plots are especially useful in hemodynamic assessment [8]. A pressure-volume (P-V) loop over a complete cardiac cycle is indicative of the force of myocardial contraction. The integral of the region enclosed by the P-V loop equals the amount of work performed by the heart (usually the left ventricle specifically) in one cycle:

$$
\int_{V_{\min }}^{V_{\max }} \int_{P_{\min }}^{P_{\max }} f(P, V) \mathrm{d} P \mathrm{~d} V=W
$$

where $V_{\min }, V_{\max }$ and $P_{\min }, P_{\max }$ are the minimum and maximum left ventricular volumes and pressures, respectively, and $W$ is the work performed. If isovolumic contraction and relaxation of the left ventricle is assumed, the double integral of equation (8) simplifies to:

$$
\int_{\mathrm{ESV}}^{\mathrm{EDV}} P \mathrm{~d} V=W
$$

where ESV and EDV are the end-systolic and end-diastolic volumes, respectively, and $P=f(V)=f_{1}(V)-f_{2}(V)$, in which $f_{1}(V)$ is a function representing left ventricular ejection and $f_{2}(V)$ left ventricular filling.

Since there is no convenient method to directly measure total or regional left 
ventricular volume in vivo, an approximation using cardiac wall segment length, wall thickness, or volume diameter is often used. As shown in Fig. 7, an LVP-segment length loop is displayed and the integral inside this loop has been calculated by moving the cursor, currently at the right of the plot, to a point inside the loop. The program computes the integral of equation (8) by counting the number of graphic pixels inside the loop, normalizing this number for the specific graphics resolution, and calibrating the value to $\mathrm{mmHg} \cdot \mathrm{mm}$. Cumulative loop area may be calculated if there is more than 1 closed loop per plot and areas may also be subtracted from the total. The loop integral

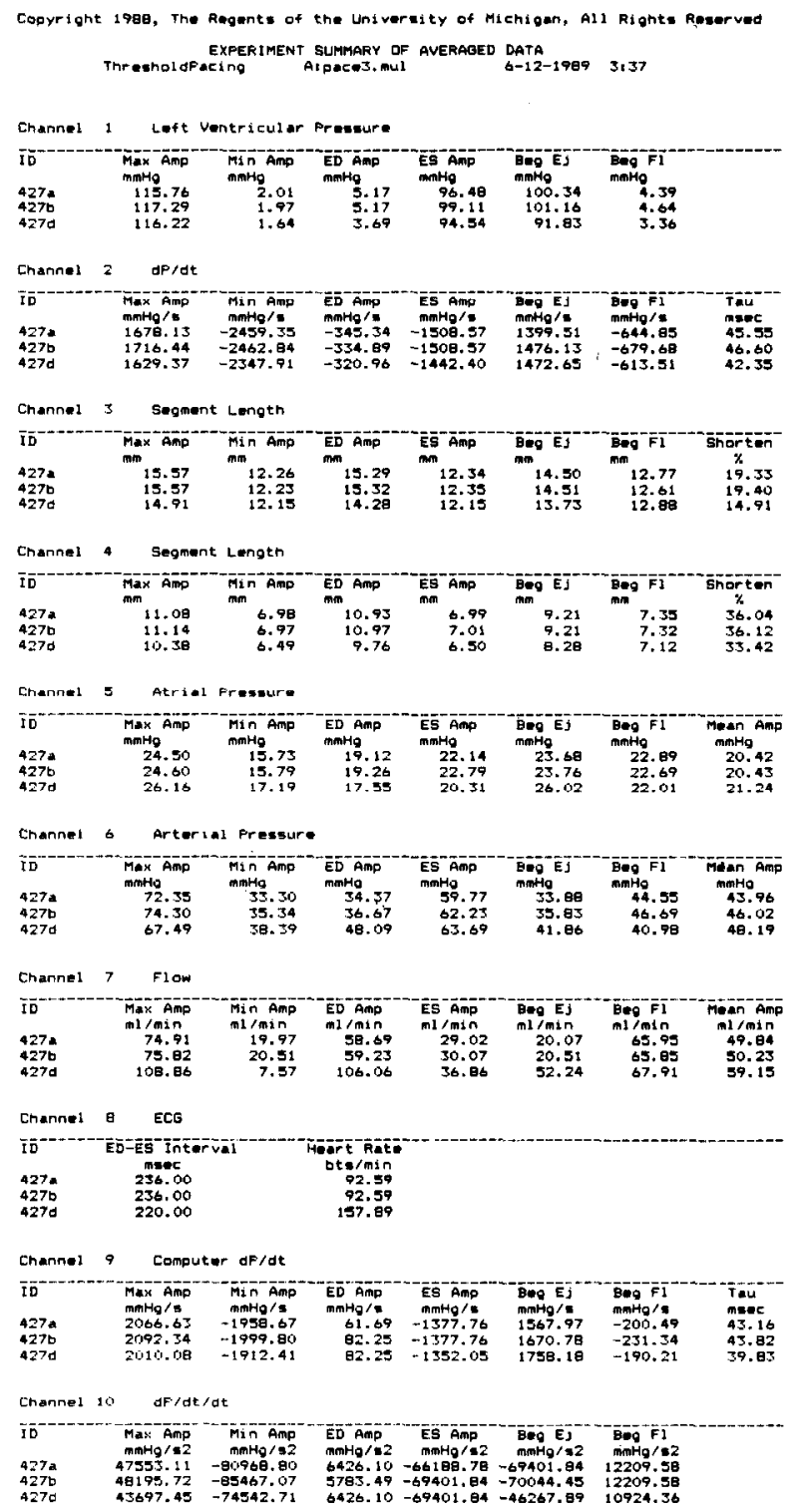

Fig. 5. Experimental summary file of averaged data results. At the top is the experiment title (threshold pacing), followed by the filename, date and time. Appropriate data for each channel are given below this information. The channels are as designated (e.g. channel 1 is left ventricular pressure). There are three entries (rows) for each channel which represent these separate sampling intervals and each have an entry ID designation. The data from each entry are based upon one averaged beat for that sampling interval. Abbreviations are as follows: Max ampmaximum amplitude over averaged beat, Min amp-minimum amplitude, ED amp-amplitude at end-diastole, ES amp-amplitude at end-systole, Beg Ej-amplitude at begin-ejection, Beg f-amplitude at begin-filling, Mean amp-point-by-point mean amplitude, Tau-ventricular isovolumic relaxation time constant, Shorten - percent increase from end-systole to end-diastole, ED-ES interval-interval from end-disastole to end-systole. This file and print-out provide a convenient summary of an experimental protocol. 


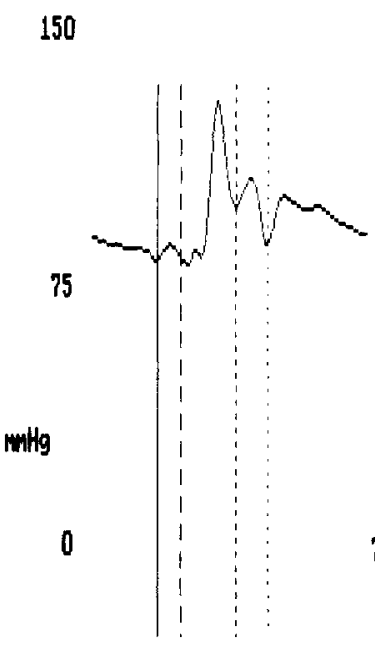

\title{
Channel 6 Arterial Pressure
}

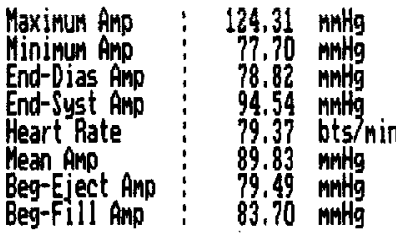

756 MS

\author{
(- or - ) to toggle between channels \\ 's to re-scale \\ ' $p$ ' to print the screen \\ 'ESC' to return to main menu \\ 'C' to rewove help
}

Fig. 6. Average beat scaled plot with beat markers and parameters. Average carotid arterial pressure shown here. Vertical beat marker lines from left are end-diastole, begin-ejection, endsystole, and begin-filling. On-screen help information is located at the bottom right corner of the figure. Abbreviations: Amp-amplitude, Dias-diastole, Syst-systole, Eject-ejection,

$$
\text { Fill-filling. }
$$

result is displayed at the bottom right corner of Fig. 7. This calculation can be used for relative comparisons of the amount of regional work performed.

\section{RESULTS}

The program has been extensively tested in a cardiovascular animal laboratory using the following canine signals: ECG (Lead II), LVP obtained from a solid state micromanometer, arterial pressures and left and right atrial pressures obtained via fluid filled

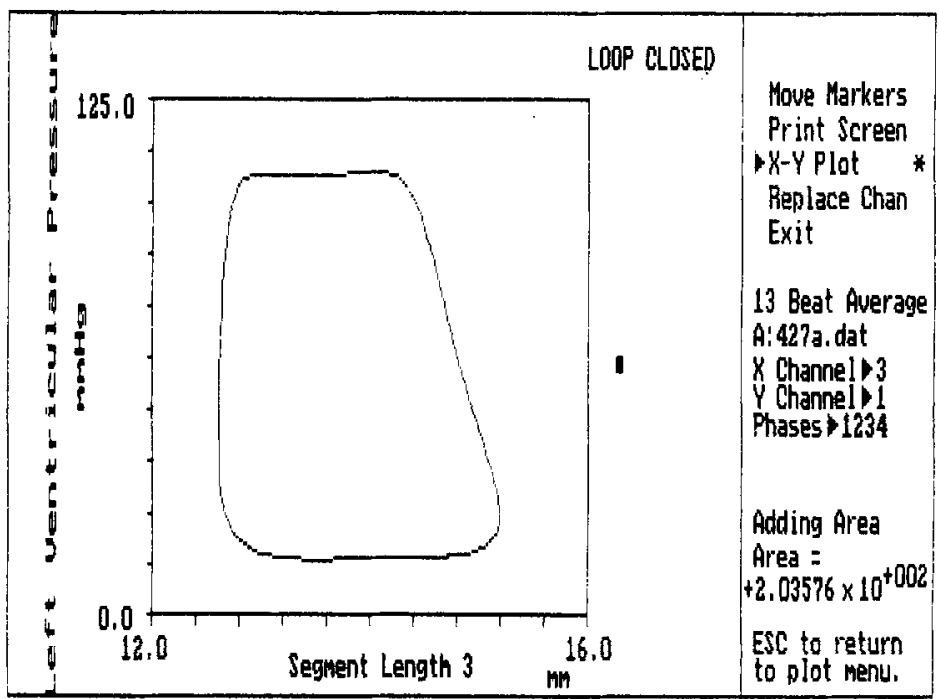

Fig. 7. Pressure-cardiac wall segment length loop display on two dimensional $(\mathrm{X}-\mathrm{Y})$ plot. Segment length from channel 4 is indicated on the $X$ axis in $\mathrm{mm}$, while left ventricular pressure appears along the $\mathrm{Y}$ axis in $\mathrm{mmHg}$. The information on the right indicates that the plot is based on an 11 beat average, channels 4 and 1 were selected as the $X$ and $Y$ channels, and all 4 phases of the heart cycle were selected for plotting. (Phase $1=$ begin-filling to end-diastole, Phase $2=$ end-diastole to begin-ejection, Phase $3=$ begin-ejection to end-systole, Phase $4=$ end-systole to begin-filling.) The area inside the loop has been calculated automatically and is displayed in the lower right corner (units: $\mathrm{mmHg} \cdot \mathrm{mm}$ ). 
catheters connected to strain-gauge transducers, blood flow obtained via electromagnetic flow probes, and cardiac wall segment length measurements obtained via implanted piezoelectric crystals and sonomicrometer.

In a series of experiments designed to prove the accuracy and reproducibility of the computer algorithms, eight open-chest dogs were monitored for LVP, carotid arterial pressure, and myocardial segment length during a baseline state in which ventricular pacing was the only intervention. These signals, including an analog $\mathrm{d} P / \mathrm{d} t$ and ECG, were recorded on FM magnetic tape (Hewlett Packard Model 3968A) at a speed of $15 / 32 \mathrm{in} / \mathrm{sec}$ (passband: DC- $156 \mathrm{~Hz}$ ). Heart rates of eight dogs ranged from 79 to 166 beats $/$ min (mean $=137$ beats $/ \mathrm{min}$ ). One $10 \mathrm{sec}$ segment of data from each dog was analysed for comparison of 3 analysis methods. The 3 methods were: (1) manual, byhand analysis using an 8 channel chart writer (Gould Model 1800S) and an experienced laboratory technician (paper speed $=100 \mathrm{~mm} / \mathrm{sec}$ ); (2) averaged beat computer analysis as described above; and (3) beat-by-beat mean computer analysis as described above. The digitization rate for computer analysis was $250 \mathrm{~Hz} /$ channel. The amplitudes of LVP, carotid arterial pressure, and segment length at end-diastole (ED), end-systole (ES), begin-ejection (BE), and begin-filling (BF) were compared for each of the 3 methods. Computer default values (Table 1), with the exception that the digitized analog $\mathrm{d} P / \mathrm{d} t$ (channel 2) was used to detect begin-ejection and end-systole, were used for all computer analyses. All cardiac cycle amplitudes were reported as automatically placed by the computer algorithms (i.e. there was no user movement of marker positions). For manual analysis, the technician was instructed to also use these computer defaults for definition of fiducial marks (i.e. $E D=R$-wave peak location, $E S=10$ msec before $\mathrm{d} P / \mathrm{d} t_{\min }$, $\mathrm{BE}=10 \mathrm{msec}$ after $\mathrm{d} P / \mathrm{d} t_{\max }, \mathrm{BF}=$ time of first occurrence of LVEDP during ventricular relaxation). Manual analysis was blinded from and was performed before all computer analyses.

The results of the 3 data analysis methods, reported with appropriate precision, are shown in Table 2. This table compares the 3 methods of analysis at each marker for each signal for the 8 experiments (dogs). With the exception of only 2 data points, a close correlation of the 3 methods is apparent for all signals at each marker. Table 2 shows that the LVP computer average beat begin-ejection amplitude for experiment 4 $(100.17 \mathrm{mmHg})$ differs markedly from the by-hand and computer beat-by-beat means ( 87 and $92.69 \mathrm{mmHg}$, respectively). Also in Table 2, the arterial pressure average beat begin-filling amplitude for experiment 1 is $87.45 \mathrm{mmHg}$ while the by-hand and computer beat-by-beat means are 103 and $104.76 \mathrm{mmHg}$, respectively.

Least-squares linear regression was performed comparing each of the methods with the other (Tables 3 through 5). All correlations $(R)$ are above 0.90 and most are greater than 0.99 . Also, the line equations (as given by slope and int) are generally close to the line of identity, indicating close agreement between methods. The lowest correlations in Tables 3 and 4 occur for LVP end-diastole $(R=0.92)$ and begin-filling $(R=0.91)$. Other relatively low correlations in these two tables occur for LVP begin-ejection and carotid arterial pressure begin-filling, but only in Table 3 which compares manual and average beat amplitude calculations. Table 5 , which compares the two computer methods, shows high correlations with $R=1.00$ for all but 2 regressions. These, again, occur for LVP begin-ejection $(R=0.97)$ and arterial pressure begin-filling $(R=0.94)$.

\section{Methods}

\section{DISCUSSION}

Of the digital filter types considered for implementation in the program, an FIR filter was chosen because of its ability to be designed with linear phase which insures that the time-domain signal representations will align correctly. Several digital derivative approximations were also examined for incorporation in the program. The 3-point Lagrange method was chosen based on the results by Marble et al. [3]. In this paper, 5 digital algorithms commonly used in computing the derivative of left ventricular pressure were 
Table 2. Analysis methods comparison

\begin{tabular}{|c|c|c|c|c|c|c|c|c|}
\hline & Exp 1 & $\operatorname{Exp} 2$ & $\operatorname{Exp} 3$ & Exp 4 & Exp 5 & Exp 6 & $\operatorname{Exp} 7$ & Exp 8 \\
\hline \multicolumn{9}{|c|}{ Left ventricular pressure } \\
\hline $\begin{array}{l}\text { Hand-ED } \\
\text { Ave-ED } \\
\text { Beat-ED }\end{array}$ & $\begin{array}{l}13 \\
12.07 \\
12.05\end{array}$ & $\begin{array}{l}3 \\
3.73 \\
3.71\end{array}$ & $\begin{array}{l}8 \\
5.47 \\
5.46\end{array}$ & $\begin{array}{l}8 \\
6.62 \\
6.61\end{array}$ & $\begin{array}{l}4 \\
6.34 \\
6.33\end{array}$ & $\begin{array}{l}5 \\
3.72 \\
3.69\end{array}$ & $\begin{array}{l}4 \\
5.21 \\
5.20\end{array}$ & $\begin{array}{l}14 \\
13.83 \\
14.18\end{array}$ \\
\hline $\begin{array}{l}\text { Hand-ES } \\
\text { Ave-ES } \\
\text { Beat-ES }\end{array}$ & $\begin{array}{l}86 \\
85.15 \\
87.46\end{array}$ & $\begin{array}{l}49 \\
53.02 \\
52.76\end{array}$ & $\begin{array}{l}46 \\
47.37 \\
47.10\end{array}$ & $\begin{array}{l}61 \\
63.90 \\
63.93\end{array}$ & $\begin{array}{l}70 \\
73.82 \\
74.21\end{array}$ & $\begin{array}{l}86 \\
86.98 \\
86.44\end{array}$ & $\begin{array}{l}97 \\
96.64 \\
96.75\end{array}$ & $\begin{array}{l}84 \\
79.13 \\
80.08\end{array}$ \\
\hline $\begin{array}{l}\text { Hand-BE } \\
\text { Ave-BE } \\
\text { Beat-BE }\end{array}$ & $\begin{array}{l}128 \\
127.01 \\
125.73\end{array}$ & $\begin{array}{l}107 \\
113.55 \\
113.50\end{array}$ & $\begin{array}{l}100 \\
101.32 \\
99.22\end{array}$ & $\begin{array}{c}87 \\
100.17 \\
92.69\end{array}$ & $\begin{array}{l}86 \\
88.49 \\
91.31\end{array}$ & $\begin{array}{l}116 \\
113.03 \\
115.65\end{array}$ & $\begin{array}{l}107 \\
105.93 \\
106.57\end{array}$ & $\begin{array}{l}93 \\
90.88 \\
90.20\end{array}$ \\
\hline $\begin{array}{l}\text { Hand-BF } \\
\text { Ave-BF } \\
\text { Beat-BF }\end{array}$ & $\begin{array}{l}13 \\
12.01 \\
12.83\end{array}$ & $\begin{array}{l}3 \\
3.17 \\
3.39\end{array}$ & $\begin{array}{l}8 \\
5.07 \\
5.08\end{array}$ & $\begin{array}{l}8 \\
6.42 \\
6.23\end{array}$ & $\begin{array}{l}4 \\
6.34 \\
6.21\end{array}$ & $\begin{array}{l}5 \\
3.46 \\
3.19\end{array}$ & $\begin{array}{l}4 \\
4.39 \\
4.55\end{array}$ & $\begin{array}{l}14 \\
13.50 \\
13.13\end{array}$ \\
\hline \multicolumn{9}{|c|}{ Myocardial segment length } \\
\hline $\begin{array}{l}\text { Hand-ED } \\
\text { Ave-ED } \\
\text { Beat-ED }\end{array}$ & $\begin{array}{l}14.6 \\
14.52 \\
14.52\end{array}$ & $\begin{array}{l}16.9 \\
16.99 \\
16.99\end{array}$ & $\begin{array}{l}15.1 \\
16.12 \\
16.12\end{array}$ & $\begin{array}{l}16.4 \\
16.32 \\
16.32\end{array}$ & $\begin{array}{l}12.1 \\
12.05 \\
12.05\end{array}$ & $\begin{array}{l}8.7 \\
8.76 \\
8.76\end{array}$ & $\begin{array}{l}15.2 \\
15.30 \\
15.30\end{array}$ & $\begin{array}{l}10.4 \\
10.36 \\
10.37\end{array}$ \\
\hline $\begin{array}{l}\text { Hand-ES } \\
\text { Ave-ES } \\
\text { Beat-ES }\end{array}$ & $\begin{array}{l}12.4 \\
12.37 \\
12.37\end{array}$ & $\begin{array}{l}13.7 \\
13.71 \\
13.71\end{array}$ & $\begin{array}{l}13.4 \\
14.38 \\
14.38\end{array}$ & $\begin{array}{l}13.9 \\
13.77 \\
13.77\end{array}$ & $\begin{array}{l}9.3 \\
9.29 \\
9.26\end{array}$ & $\begin{array}{l}6.6 \\
6.70 \\
6.70\end{array}$ & $\begin{array}{l}12.4 \\
12.34 \\
12.32\end{array}$ & $\begin{array}{c}10.1 \\
10.00 \\
9.99\end{array}$ \\
\hline $\begin{array}{l}\text { Hand-BE } \\
\text { Ave-BE } \\
\text { Beat-BE }\end{array}$ & $\begin{array}{l}13.4 \\
13.33 \\
13.33\end{array}$ & $\begin{array}{l}15.2 \\
15.18 \\
15.17\end{array}$ & $\begin{array}{l}14.1 \\
15.07 \\
15.08\end{array}$ & $\begin{array}{l}15.6 \\
15.42 \\
15.46\end{array}$ & $\begin{array}{l}10.5 \\
10.47 \\
10.44\end{array}$ & $\begin{array}{l}7.6 \\
7.63 \\
7.63\end{array}$ & $\begin{array}{l}15.5 \\
14.47 \\
14.46\end{array}$ & $\begin{array}{l}11.1 \\
11.11 \\
11.10\end{array}$ \\
\hline $\begin{array}{l}\text { Hand-BF } \\
\text { Ave-BF } \\
\text { Beat-BF }\end{array}$ & $\begin{array}{l}14.1 \\
14.45 \\
13.96\end{array}$ & $\begin{array}{l}14.0 \\
14.25 \\
14.22\end{array}$ & $\begin{array}{l}13.2 \\
14.20 \\
14.21\end{array}$ & $\begin{array}{l}13.2 \\
13.24 \\
13.23\end{array}$ & $\begin{array}{l}9.5 \\
9.67 \\
9.69\end{array}$ & $\begin{array}{l}7.2 \\
7.19 \\
7.28\end{array}$ & $\begin{array}{l}12.6 \\
12.74 \\
12.73\end{array}$ & $\begin{array}{l}9.5 \\
9.52 \\
9.51\end{array}$ \\
\hline \multicolumn{9}{|c|}{ Carotid arterial pressure } \\
\hline $\begin{array}{l}\text { Hand-ED } \\
\text { Ave-ED } \\
\text { Beat-ED }\end{array}$ & $\begin{array}{l}94 \\
93.33 \\
93.31\end{array}$ & $\begin{array}{l}106 \\
106.43 \\
106.41\end{array}$ & $\begin{array}{l}86 \\
85.43 \\
85.42\end{array}$ & $\begin{array}{l}115 \\
116.12 \\
116.10\end{array}$ & $\begin{array}{l}69 \\
69.51 \\
69.49\end{array}$ & $\begin{array}{l}91 \\
93.88 \\
93.37\end{array}$ & $\begin{array}{l}94 \\
94.72 \\
94.72\end{array}$ & $\begin{array}{l}82 \\
82.93 \\
82.88\end{array}$ \\
\hline $\begin{array}{l}\text { Hand-ES } \\
\text { Ave-ES } \\
\text { Beat-ES }\end{array}$ & $\begin{array}{l}107 \\
106.98 \\
107.83\end{array}$ & $\begin{array}{l}113 \\
113.12 \\
113.83\end{array}$ & $\begin{array}{l}105 \\
104.65 \\
104.19\end{array}$ & $\begin{array}{l}125 \\
125.33 \\
125.12\end{array}$ & $\begin{array}{l}89 \\
88.99 \\
88.53\end{array}$ & $\begin{array}{l}107 \\
107.00 \\
105.87\end{array}$ & $\begin{array}{l}121 \\
119.93 \\
119.97\end{array}$ & $\begin{array}{l}99 \\
100.41 \\
100.25\end{array}$ \\
\hline $\begin{array}{l}\text { Hand-BE } \\
\text { Ave-BE } \\
\text { Beat-BE }\end{array}$ & $\begin{array}{l}86 \\
85.01 \\
85.11\end{array}$ & $\begin{array}{l}109 \\
111.31 \\
111.63\end{array}$ & $\begin{array}{l}91 \\
91.22 \\
90.57\end{array}$ & $\begin{array}{l}104 \\
105.19 \\
104.92\end{array}$ & $\begin{array}{l}71 \\
70.78 \\
69.83\end{array}$ & $\begin{array}{l}89 \\
91.70 \\
91.75\end{array}$ & $\begin{array}{l}94 \\
94.27 \\
94.25\end{array}$ & $\begin{array}{l}77 \\
77.70 \\
78.01\end{array}$ \\
\hline $\begin{array}{l}\text { Hand-BF } \\
\text { Ave-BF } \\
\text { Beat-BF }\end{array}$ & $\begin{array}{c}103 \\
87.45 \\
104.76\end{array}$ & $\begin{array}{l}105 \\
109.13 \\
108.33\end{array}$ & $\begin{array}{l}83 \\
80.74 \\
81.38\end{array}$ & $\begin{array}{l}119 \\
124.70 \\
124.50\end{array}$ & $\begin{array}{l}69 \\
66.56 \\
68.06\end{array}$ & $\begin{array}{l}89 \\
92.85 \\
93.50\end{array}$ & $\begin{array}{l}102 \\
104.52 \\
104.22\end{array}$ & $\begin{array}{l}85 \\
84.53 \\
85.10\end{array}$ \\
\hline
\end{tabular}

Abbreviations: $\operatorname{Exp}=$ experiment, Hand = manual analysis, Ave $=$ computer-averaged beat analysis, Beat $=$ computer beat-by-beat mean analysis, $\mathrm{ED}=$ end-diastole, $\mathrm{ES}=$ end-systole, $\mathrm{BE}=$ begin-ejection, $\mathrm{BF}=$ begin-filling

Units: $L V P=m m I g, M S L=m m, C A P=m m H g$.

Table 3. Regression analysis of manual vs average beat amplitude calculation

\begin{tabular}{|c|c|c|c|c|c|c|c|c|c|c|c|c|}
\hline \multirow[b]{2}{*}{ Signal } & \multicolumn{3}{|c|}{ End-diastole } & \multicolumn{3}{|c|}{ End-systole } & \multicolumn{3}{|c|}{ Begin-ejection } & \multicolumn{3}{|c|}{ Begin-filling } \\
\hline & Slope & Int & $R$ & Slope & Int & $R$ & Slope & Int & $R$ & Slope & Int & $R$ \\
\hline LVP & 1.07 & -0.11 & 0.92 & 1.10 & -7.94 & 0.99 & 1.06 & -7.87 & 0.93 & 1.03 & 0.44 & 0.91 \\
\hline MSL & 0.97 & 0.29 & 0.99 & 0.95 & 0.41 & 0.99 & 1.00 & -0.03 & 0.98 & 0.93 & 0.54 & 0.99 \\
\hline CAP & 1.01 & 0.40 & 1.00 & 0.98 & 2.37 & 1.00 & 1.05 & -4.22 & 1.00 & 1.09 & -8.54 & 0.93 \\
\hline
\end{tabular}

Abbreviations: $L V P=$ left ventricular pressure, $M S L=$ myocardial segment length, $C A P=$ carotid arterial pressure, Slope $=$ slope of least-squares linear regression line, Int = intercept of least-squares linear regression line, $R=$ correlation coefficient of linear regression.

Units: LVP Int $=\mathrm{mmHg}$, MSL Int $=\mathrm{mm}$, CAP Int $=\mathrm{mmHg}$. 
Table 4. Regression analysis of manual vs beat-by-beat average amplitude calculation

\begin{tabular}{|c|c|c|c|c|c|c|c|c|c|c|c|c|}
\hline \multirow[b]{2}{*}{ Signal } & \multicolumn{3}{|c|}{ End-diastole } & \multicolumn{3}{|c|}{ End-systole } & \multicolumn{3}{|c|}{ Begin-ejection } & \multicolumn{3}{|c|}{ Begin-filling } \\
\hline & Slope & Int & $R$ & Slope & Int & $R$ & Slope & Int & $R$ & Slope & Int & $R$ \\
\hline LVP & 1.04 & 0.05 & 0.92 & 1.08 & -6.79 & 0.99 & 1.06 & -7.81 & 0.97 & 1.01 & 0.57 & 0.91 \\
\hline MSL & 0.97 & 0.28 & 0.99 & 0.95 & 0.43 & 0.99 & 1.00 & 0.02 & 0.98 & 0.96 & 0.25 & 0.99 \\
\hline CAP & 1.01 & 0.26 & 1.00 & 0.99 & 0.81 & 1.00 & 1.07 & -6.26 & 1.00 & 1.13 & -10.64 & 1.00 \\
\hline
\end{tabular}

Abbreviations: Same as Table 3.

Units: Same as Table 3.

compared experimentally. Of the algorithms examined, the 3-point Lagrange derivative resulted in the lowest quantization error of the left ventricular pressure signal when 12bit $\mathrm{A} / \mathrm{D}$ resolution was implemented.

Of the signals typically monitored in a cardiovascular laboratory, the ECG is most often used and is usually the best choice for a beat averaging trigger. Specifically, the QRS complex peak serves as the fiducial point. The QRS peak minimizes signal averaging degradation due to trigger jitter [9], since it is relatively consistent in position beat-to-beat during normal rhythm and contains relatively high frequency components. The ECG may be differentiated and/or filtered to better isolate the QRS if T-waves, pacemaker depolarization pulses, or noise are high enough in amplitude and frequency content to be falsely detected as QRS complexes. If an ECG signal is not available, $\mathrm{d} P / \mathrm{d} t_{\max }$ or $\mathrm{d} P / \mathrm{d} t_{\min }$ may be used as the signal averaging trigger. However, their peak positions show more variability in the cardiac cycle than the QRS peak. Also, it is more difficult to detect, and therefore discard, abnormal cardiac depolarizations using $\mathrm{d} P / \mathrm{d} t$.

The end of diastole is determined using one of two methods, chosen by the investigator, as noted in the program description. The first and default method which positions end-diastole at the QRS complex peak of the ECG is a technique used by many investigators $[10,11]$. There may be instances in which the ECG is not monitored or there may be problems dependent upon the QRS morphology and lead selection (e.g. confusion as to whether to choose the positive or negative peak of the QRS). In these cases an alternate means of determining end-diastole, not previously described in the literature, has been implemented. This empirically derived method locates the first significant, positive $\mathrm{d}^{2} P / \mathrm{d} t^{2}$ peak of the cycle and labels it end-diastole. Many researchers have determined end-diastole using a slope threshold of the initial rising edge of $\mathrm{d} P / \mathrm{d} t$ [12-14]. We have tried this technique, but were unable to determine a suitable slope threshold for all of the data tested. Other investigators have used the zero crossing prior to the initial rise in $\mathrm{d} P / \mathrm{d} t[15]$, but we found this point to be too variable in position from beat to beat. Still others determined end-diastole by the nadir of LVP beteen atrial and ventricular systole $[16,17]$. However, since animals in the laboratory frequently have AV syncrony interrupted, through AV node ablation and/or pacing, this method was considered ineffective. Our new technique using $\mathrm{d}^{2} P / \mathrm{d} t^{2}$ is not dependent upon a slope threshold and has given reliable, reproducible results on the data tested thus far.

Most investigators use $\mathrm{d} P / \mathrm{d} t_{\min }$ to locate end-systole [11, 13-15, 17]. However, there is controversy regarding the position of end-systole relative to this minimum amplitude. Placement ranges anywhere from $\mathrm{d} P / \mathrm{d} t_{\min }$ itself to $20 \mathrm{msec}$ prior to $\mathrm{d} P / \mathrm{d} t_{\min }$. We chose $10 \mathrm{msec}$ prior to $\mathrm{d} P / \mathrm{d} t_{\min }$ as the default value based upon the laboratory experience of

Table 5. Regression analysis of average beat vs beat-by-beat average amplitude calculation

\begin{tabular}{|c|c|c|c|c|c|c|c|c|c|c|c|c|}
\hline \multirow[b]{2}{*}{ Signal } & \multicolumn{3}{|c|}{ End-diastole } & \multicolumn{3}{|c|}{ End-systole } & \multicolumn{3}{|c|}{ Begin-ejection } & \multicolumn{3}{|c|}{ Begin-filling } \\
\hline & Slope & Int & $R$ & Slope & Int & $R$ & Slope & Int & $R$ & Slope & Int & $R$ \\
\hline LVP & 0.98 & 0.15 & 1.00 & 0.98 & 1.23 & 1.00 & 0.94 & 7.10 & 0.97 & 0.98 & 0.13 & 1.00 \\
\hline MSL & 1.00 & -0.01 & 1.00 & 1.00 & 0.02 & 1.00 & 1.00 & 0.04 & 1.00 & 1.03 & -0.31 & 1.00 \\
\hline CAP & 1.00 & -0.06 & 1.00 & 1.01 & -1.60 & 1.00 & 1.02 & -1.98 & 1.00 & 0.92 & 9.90 & 0.94 \\
\hline
\end{tabular}

Abbreviations: Same as Table 3.

Units: Same as Table 3. 
one of us (JMN). Note that at a sampling rate of $250 \mathrm{~Hz}, 10 \mathrm{msec}$ is rounded to $12 \mathrm{msec}$ because of the $4 \mathrm{msec}$ sampling interval.

When aortic and pulmonary artery pressures are not measured (which we assume), $\mathrm{d} P / \mathrm{d} t_{\max }$ is generally used as a marker for determining begin-ejection $[15,18]$. Researchers specify anywhere from 0 to $20 \mathrm{msec}$ following $\mathrm{d} P / \mathrm{d} t_{\max }$ as begin-ejection. We chose $10 \mathrm{msec}$ after $\mathrm{d} P / \mathrm{d} t_{\max }$ as a compromise default value. Again, at $250 \mathrm{~Hz}$, $10 \mathrm{msec}$ is rounded to $12 \mathrm{msec}$.

Researchers, in lieu of an atrial pressure signal, usually mark begin-filling on the negative, relaxation slope of LVP at the point which equals LVEDP [19-21]. Locating the beginning of filling is important, since it marks the end-point for the calculation of $T$. Some laboratories $[22,23]$ have used a point $5 \mathrm{mmHg}$ above LVEDP to terminate isovolumic relaxation, so an option of specifying a number of $\mathrm{mmHg}$ above or below LVEDP has been included.

\section{Results}

The results from $10 \mathrm{sec}$ data segments from 8 dogs which were analysed using hand measurement and the two computer methods were listed in Table 2. Least-squares linear regressions comparing each of the methods with another were reported in Tables 3 through 5. Ovcrall, Tables 3, 4 and 5 showed excellent correlations and least-square lines were close to the line of identity. Therefore, Tables 3 and 4 indicate that the computer algorithms are indeed accurate. Table 5 indicates that a single averged beat for each channel may be used for cardiac cycle analysis to accurately replace computer beat-bybeat feature averaging. Table 5 also implies the greater precision of computer methods over manual analysis.

Tables 3 and 4, which compare each of the computer methods to manual analysis, have relatively low correlations for LVP end-diastole and begin-filling. These values are probably due to the low amplitudes at end-diastole and begin-filling which cause problems for manual measurement which lacks the precision of the computer methods. Correlations between the computer methods of LVP at ED and BF were both 1.00 indicating that the error was most likely in the by-hand measurement.

The two other low correlations in Table 3, LVP at begin-ejection and carotid arterial pressure at begin-filling, appear to be due to two outliers in the averaged beat calculations. The averaged beat, begin-ejection amplitude of LVP for experiment 4 (Table 2) of $100.17 \mathrm{mmHg}$ differs markedly from the other two methods. This one difference lowers the LVP begin-ejection correlation to its value of 0.93 . This discrepancy appears to be due to amplitude and position variability in $\mathrm{d} P / \mathrm{d} t_{\max }$ in this data segment. Because of the steep, positive slope at begin-ejection, user movement of the average beat begin-ejection marker back only $8 \mathrm{msec}$ lowers the LVP to $92.88 \mathrm{mmHg}$ at this point, a value very close to the beat-by-beat mean of $92.69 \mathrm{mmHg}$.

Also, the averaged beat, begin-filling amplitude of arterial pressure for experiment 1 (Table 2) of $87.45 \mathrm{mmHg}$ differs substantially from the other two values. This lowers the arterial pressure begin-filling correlation to 0.93 . The difference in results is due to the placement of the begin-filling marker approximately $40 \mathrm{msec}$ following its typical beatby-beat location. It simply took the average beat LVP waveform that much longer to reach a value which was less than or equal to LVEDP. This occurred because enddiastolic LVP was close to minimum pressure and the period of ventricular filling was rather constant in amplitude. Since carotid pressure is rapidly changing during this period, its resulting averaged beat amplitude was approximately $17 \mathrm{mmHg}$ lower than the other 2 methods. Some investigators [22,23] have set the begin-filling position at LVEDP $+5 \mathrm{mmHg}$ which would help alleviate this problem. Comparisons between the 2 computer algorithms (Table 5) indicate that the 2 discrepancies in correlation are indeed due to these averaged beat outliers since: (1) the only correlations below 1.00 are for LVP begin-ejection $(R=0.97)$ and carotid pressure begin-filling $(R=0.94)$; and (2) the only amplitudes in these 2 comparisons deviating by more than $3 \%$ are these values from experiments 1 and 4 which differ by $17 \%$ and $8 \%$, respectively. 
Signal averaging of cardiac cycles before placement of cardiac cycle markers would be a valuable, efficient method of analysis for a cardiovascular laboratory. The alternative method of computer analysis, involving beat-by-beat placement of fiducial markers, requires user validation and possible movement of numerous markers by the investigator to their correct positions. This usually involves checking 8-30 beats in a $10 \mathrm{sec}$ data segment. Although, the computer package presented here allows this type of analysis, the preferred method for the investigator would be the validation and possible correction of cardiac cycle markers on a single averaged beat. The analysis presented here, comparing averaged beat and beat-by-beat average cycle parameters, demonstrates that signal averaging gives results which are nearly identical to individual beat feature averaging for LVP, segment length, and carotid pressure. This is demonstrated in Table 5 by the very high $R$ values and the close correspondence of the line equations to the line of identity. Any trigger jitter [9] present in beat-to-beat QRS position did not appear to be a problem affecting the averaging and resultant cardiac cycle amplitudes. Indeed, whenever appreciable noise is present in the signal, beat averaging should be the preferred method, since it will reduce the degree of random noise present.

Tables 3 and 4 demonstrate that the computer methods compare well to hand analysis and we therefore infer, due to the blinded nature of the analysis, that the computer algorithms are accurate in their determination of the cardiac cycle markers, signal calibration, and amplitude calculation. The manual analysis is, of course, much less precise than the computer analyses, but provides an overall indication of the computer algorithms' accuracy by comparison to an independent method. Myocardial segment length shows especially high correlations and without exception $(R>0.98)$. This is probably due to the low frequency content of the signal which causes the reporting of similar results even if the cycle marker positions differ by an appreciable number of msec.

\section{Comparison to other systems}

Many current laboratory systems which purport to be automatic are, in general, data loggers with simple signal acquisition and data base capabilities. Other physiologic analysis software may require extensive user programming to obtain the desired calculated parameters.

The system described here was compared with 4 similar cardiovascular analysis packages. Three of these systems are commercially available: The Digital Acquisition, Analysis and Archiving System (Po-Ne-Mah, Inc., Storrs, CT), The Blood Pressure Analysis System (Gould, Inc., Cleveland, $\mathrm{OH}$ ), and The Hemodynamics Analysis System (Buxco, Inc., Sharon, CT). The fourth computer analysis system is one by Farrell and Bruce [24] described in the literature. These 4 systems provide the investigator with detailed cardiovascular signal information and, therefore, serve as a fair comparison to the system described here.

The Po-Ne-Mah system, although based on the IBM-PC/AT, requires an expensive coprocessor, more extensive secondary storage, and more elaborate graphics peripherals than our system. The data acquisition is continuous and data are reported in real-time at specified time intervals. (With our program, data are acquired when requested and analysed post-acquisition.) The Po-Ne-Mah system calculated derived parameters which are not calculated by the system described here and because of the high quality graphics hardware, many plots may be specified on the same screen. Although these are valuable features, our program analyses the same signals and reports all parameters which are most common and of most importance to the researcher using less expensive hardware. In addition, our software has several significant advantages. Namely, the system we describe:

(1) Automatically discards premature, escape, and aberrant beats in averaged beat analysis.

(2) Filters and differentiates after signal digitization.

(3) Allows the operator to correct the position of misaligned beat markers. 
(4) Displays an averaged beat $\mathrm{X}-\mathrm{Y}$ plot and calculates the average plot area (e.g. pressure-volume loop area).

The first 3 advantages listed here are, at times, crucial to measurement accuracy and the fourth is a valuable analysis tool. The Po-Ne-Mah system does not detect or discard premature, escape or aberrant beats. It only filters and differentiates signals as they are digitized which does not allow data to be manipulated after it has been stored. This is a serious limitation for retrospective data analysis. Signal averaged beats are not computed and beat marker positions may not be manually corrected if found to be in error.

The Gould Blood Pressure Analysis software is also designed for the IBM-PC/AT. This system is more limited than either the system described here or that of Po-Ne-Mah. It is designed primarily for measuring aortic pressures, left ventricular pressures, and heart rate. There are no provisions for measuring wall or volume dimensions or blood flow, and only a limited number of parameters are calculated. Also, there is no capability for X-Y data plotting. Averaged beat results are not reported and all advantages of our system over the Po-Ne-Mah system apply to the Gould analysis package as well.

The Buxco Hemodynamics Analysis System is a hardware plus software package which accepts as input: arterial blood pressure, central venous pressure, pulmonary arterial pressure, ECG, left ventricular pressure, and blood flow. All commonly required parameters, such as systolic, diastolic, and mean amplitudes, and heart rate are calculated, however, begin-ejection and begin-filling are not identified. Since this system is dependent upon its own hardware configuration, equipment and computer application flexibility is compromised. Several other features of our system are not found in the Buxco analysis package. There is no analysis for wall or volume dimensions, no premature or aberrant beat rejection, no post digitization differentiation or filtering, no operator validation or correction of beat markers, and no $\mathrm{X}-\mathrm{Y}$ plotting available.

The Farrell and Bruce cardiovascular analysis software is designed to run on an APPLE II + or IIe (with 64 Kbytes RAM) and compatible A/D board. This system samples and analyses a maximum of four channels, which may consist of pulsatile flow, pulsatile pressure, drop counter, and linear flow or pressure. Appropriate parameters are analysed; however, the selection of signals and number of channels are limited, analysis is performed only on a beat-by-beat basis, consists only of numerical results (no graphics), and user validation of correct end-diastolic and end-systolic markers is not possible. Also, end-diastole and end-systole are determined directly from pulsatile pressure $(\mathrm{d} P / \mathrm{d} t$ is not used) resulting in less accurate results. Only an approximate calibration is done manually by the investigator after the signals have been digitized. In addition, user reprogramming is required to change input parameters, such as, sampling rate and sampling duration. The Farrell and Bruce cardiovascular system is considerably limited in comparison to our system which contains only slightly more expensive hardware with much more flexibility, many additional features, and greater applicability to the majority of cardiovascular laboratories.

\section{SUMMARY}

A comprehensive system of data acquisition and analysis of multiple cardiovascular signals has been described. The software features beat-by-beat and averaged beat analysis. Signal processing functions on the analog inputs include filtering, differentiation, event detection, and digital analysis. Automated measurements are produced (at end-diastole, end-systole, begin-ejection, begin-filling, positive peak, and negative peak) and derived measurements are tabulated, including the relaxation time constant. Any combination of the ten channels (eight analog inputs plus two computer derived channels) may be plotted vs time or as $\mathrm{X}-\mathrm{Y}$ plots.

The system is completely software driven and controlled with minimal operator intervention. It has been shown to be a reliable, multipurpose tool for use in a cardiovascular laboratory and has advantages over four physiologic analysis systems. Its implementation on an inexpensive general purpose personal computer, allowing a choice 
of inexpensive peripherals, should make this cardiovascular signal analysis system valuable in a variety of laboratory settings.

Acknowledgement-J. M. Nicklas is supported by the NIH Clincial Investigator Award HL 01170.

\title{
REFERENCES
}

1. A. V. Oppenheim and R. W. Schafer, Digital Signal Processing. Prentice Hall, Englewood Cliffs, NJ (1975).

2. R. A. Gabel and R. A. Roberts, Signals and Linear Systems. Wiley New York (1980).

3. A. E. Marble, C. M. McIntyre, R. Hastings-James and C. W. Hor, A comparison of digital algorithms used in computing the derivative of left ventricular pressure, IEEE Trans. Biomed. Eng. BME-28, 524 (1981).

4. J. L. Weiss, J. W. Frederiksen and M. L. Weisfeldt, Hemodynamic determinants of the time course of fall in canine left ventricular pressure, J. clin. Invest. 58, 751 (1976).

5. W. E. Craig and J. P. Murgo, Evaluation of isovolumic relaxation in normal man duirng rest, exercise, and isoproterenol infusion, Circulation 62 (suppl. II), II-92 (1980).

6. D. S. Thompson, C. B. Waldron, D. J. Coltart, B. S. Jenkins and M. M. Webb-People, Estimation of time constant of left ventricular relaxation, Br. Heart J. 49, 250 (1983).

7. W. E. Craig, J. P. Murgo and A. Pasipoularides, Evaluation of time course of left ventricular isovolumic relaxation in humans, In Diastolic Relaxation of the Heart, W. Grossman and B. H. Lorell, Eds, pp. 125132. Martinus Nijhoff, Boston, MA (1988).

8. A. M. Katz, Physiology of the Heart. Raven Press Books, New York (1977).

9. O. Rompelman and H. H. Ros, Coherent averaging technique: A tutorial review, part 2, J. Biomed. Eng. 8, 30 (1986).

10. W. Y. W. Lew, Influence of ischemic zone size on nonischemic area function in the canine left ventricle, Am. J. Physiol. 252, H990 (1987).

11. P. D. Boudillon, W. J. Paulus, T. Serizawa and W. Grossman, Effects of verapamil on regional myocardial diastolic function in pacing-induced ischemia in dogs, Am. J. Physiol. 251, H834 (1986).

12. O. M. Hess, G. Osakada, J. F. Lavelle, K. P. Gallagher, W. S. Kemper and J. Ross, Diastolic myocardial wall stiffness and ventricular relaxation during partial and complete coronary occlusions in the conscious dog, Circ. Res. 52, 387 (1983).

13. J. M. Nicklas, L. C. Becker and B. H. Bulkley, Effects of repeated brief coronary occlusion on regional left ventricular function and dimension in dogs, Am. J. Cardiol. 56, 473 (1985).

14. M. L. Charlat, P. G. O'Neill, C. J. Hartley, R. Roberts and R. Bolli, Prolonged abnormalities of left ventricular diastolic wall thinning in the "stunned" myocardium in conscious dogs: Time course and relation to systolic function, J. Am. Coll. Cardiol. 13, 185 (1989).

15. K. P. Gallagher, T. Kumada, A. Battler, W. S. Kemper and J. Ross, Isoproterenol-induced myocardial dysfunction in dogs with coronary stenosis, Am. J. Physiol. 242, H260 (1982).

16. M. S. Visner, C. E. Arentzen, D. G. Parrish, E. V. Larson, M. J. O'Connor, A. J. Crumbley, R. J. Bache and R. W. Anderson, Effects of global ischemia on the diastolic properties of the left ventricle in the conscious dog, Circulation 71, 610 (1985).

17. S. Sasayama, K. P. Gallagher, W. S. Kemper, D. Frnaklin and J. Ross, Regional left ventricular wall thickness early and late after coronary occlusion in the conscious dog, Am. J. Physiol. 240, H293 (1981).

18. D. D. Glower, J. Schaper, J. S. Kabas, H. M. Hoffmeister, W. Schaper, J. A. Spratt, J. W. Davis and J. S. Rankin, Relation between reversal of diastolic creep and recovery of systolic function after ischemic myocardial injury in conscious dogs, Circ. Rec. 60, 850 (1987).

19. T. Kumada, J. S. Karliner, H. Pouleur, K. P. Gallagher, K. Shirato and J. Ross, Effects of coronary occlusion on early ventricular diastolic events in conscious dogs, Am. J. Physiol. 237, H542 (1979).

20. D. S. Thompson, C. B. Waldron, S. M. Juul, N. Naqvi, R. H. Swanton, D. J. Coltart, B. S. Jenkins and M. M. Webb-Peploe, Analysis of left ventricular pressure during isovolumic relaxation in coronary artery disease, Circulation 65, 690 (1982).

21. P. B. Kurnik, M. R. Courtois and P. A. Ludbrook, Diastolic stiffening induced by acute myocardial infarction is reduced by early reperfusion, J. Am. Coll. Cardiol. 12, 1029 (1988).

22. M. R. Courtois, P. B. Kurnik and P. A. Ludbrook, Sensitivity of isovolumic relaxation to hypothermia during myocardial infarction, J. Am. Coll. Cardiol. 11, 201 (1988).

23. S. Monomura, A. B. Bradley and W. Grossman, Left ventricular diastolic pressure-segment length relations and end-diastolic distensibility in dogs with coronary stenoses, Circ. Res. 55, 203 (1984).

24. A. P. Farrell and F. Bruce, Data acquisition and analysis of pulsatile signals using a personal computer: An application in cardiovascular physiology, Comput. Biol. Med. 17, 151 (1987).

\begin{abstract}
About the Author-Saul E. Greenhut was born in Detroit, Michigan in 1961. He received the B.S.E. (Engineering Science) and M.S. (Bioengineering) degrees from the University of Michigan in 1983 and 1984, respectively. He worked as Research Engineer in the Department of Surgery, Newark-Beth Israel Medical Center, Newark, NJ from 1984 to 1985, and as Computer Coordinator in the Department of Cardiology, William Beaumont Hospital, Royal Oak, MI from 1985 to 1987 . In 1987, he returned to the University of Michigan to pursue a Ph.D. degree (Bioengineering) and has served as research assistant in the Departments of Internal Medicine, and Electrical Engineering and Computer Science. His doctoral thesis is entitled Computer modelling and interpretation of paced electrocardiograms for the analysis of pacemaker failure. $\mathrm{Mr}$ Greenhut's research interests include cardiovacular modelling and signal processing. He is currently a student member of the IEEE and Engineering in Medicine and Biology Society.
\end{abstract}


About the Author-Jong W. LeE received the B.S.E.E. degree in 1977 and the M.E.E. degree in 1980 from the University of Virginia, Charlottesville, Virginia, and the Ph.D. degree in 1986 in Electrical Engineering from the University of Michigan, Ann Arbor, Michigan. From 1986 to 1989. Dr Lee was a member of the technical staff at the Bell Laboratories, Holmdel, NJ, where he was involved in various AT \& T telecommunications traffic engineering analysis and parallel signal processor system design and applications. Currently, he is with the Science Applications International Corporation, McLean, VA, where he is involved in applied research in underwater signal detection and estimation theory, as well as systems correlator/tracker.

About the Author-Joseph C. Monsur was born in Detroit, Michigan in 1958. He completed the Veterinary Technology Program at Wayne State University in 1980 , passing state and national boards to become a licensed veterinary technician. He received the B.S. degree in Animal Science from Michigan State University in 1984 . He worked as a veterinary technician at Mt. Hope and the Humane Society of Macomb County Animal Hospitals from 1980 to 1985. In 1986 he came to the University of Michigan as a Research Associate in the Cardiology research laboratories. His primary research focus is collateral and myocardial blood flow in dogs.

About the Author-John M. Nicklas was born in Pittsburgh, Pennsylvania in 1950. He received his B.S. degree from Brown University in 1972 and his M.D. degree from Johns Hopkins University School of Medicine in 1976. His post-doctoral training in Internal Medicine and fellowship in Cardiology were done at the Johns Hopkins Hospital. Dr Nicklas has been a faculty member at the University of Michigan since 1982 and is presently an Associate Professor of Internal Medicine and Director of the Coronary Intensive Care Unit at the University of Michigan Hospital. Dr Nicklas' research interests include myocardial metabolism and congestive heart failure. 